\title{
Bat-inspired Optimizer for Prediction of Anti-Viral Cure Drug of SARS-CoV-2 based on Recurrent Neural Network
}

\author{
Ismail Elansary ${ }^{1, *}$, Walid Hamdy ${ }^{1, *}$, Ashraf Darwish ${ }^{2, *}$ and Aboul Ella Hassanien ${ }^{3, *}$ \\ ${ }^{1}$ Faculty1 of Science, Port Said University, Cairo, Egypt \\ ${ }^{2}$ Faculty of Science, Helwan University, Cairo, Egypt \\ ${ }^{3}$ Faculty of Computers and Artificial Intelligence, Cairo University, Giza, Egypt. \\ *Scientific Research Group in Egypt (SRGE), http://www.egyptscience.net \\ a_rahman@yahoo.com
}

\begin{abstract}
COVID-19 is a large family of viruses that causes diseases ranging from the common cold to more severe diseases such as SARS-CoV. There are currently several attempts to create an anti-viral drug to combat the virus. The antiviral medicines could be promising treatment choices for COVID-19. Therefore, a fast strategy for drugs application that can be utilized to the patient immediately is necessary. In this context, deep learning-based architectures can be considered for predicting drug-target interactions accurately. This is due to a large amount of complicated knowledge, such as hydrophobic interactions, ionic interactions, and bonding with hydrogen. In this paper, Recurrent Neural Network (RNN) is used to build drug-target interaction prediction model to predict drug-target interactions. Bat Algorithm (BA) is used in this paper to optimize the model parameters of RNN (RNN-BA) and then using the corona virus as a target. The drug with the best binding affinity will be a potential cure for the virus. The proposed model consists of different four phases; data preparation phase, hyper-parameters optimizing phase, learning phase and fine-tuning for specific ligand subsets. The used dataset in this paper to train and evaluate the proposed model is selected from a total of 677,044 SMILES. The experimental results of the proposed model showed high level of performance in comparison with the related approaches.
\end{abstract}

Keywords: COVID-19, SARS-CoV-2, Recurrent Neural Network, Bat algorithm, drug design, Anti-Viral cure drug. 


\section{Introduction}

Research that claims Chloroquine and Remdesivir which is an HIV inhibitor has been recognized as effective candidates to control the virus (Wang et al., 2020). Innovation Pharmaceuticals is evaluating Brilacidin as a candidate treatment for the virus, CytoDyn are also examining a potential cure for the virus called leronlimab [2]. Many other research facilities are also burning the mid-night candle to develop a potential treatment for the virus. A potential cure for the virus is to have an inhibitor (Antibody) that will attach itself to the virus and prevent it from attaching to the human cell's receptors which will prevent it from spreading. Author in [3] developed a set of sample candidate drugs using deep reinforcement learning and then using the corona virus as a target, bind all the sample candidate drugs to it and find out the one with the best binding affinity. The drug with the best binding affinity will be a potential cure for the virus.

Drug repurposing is a new therapeutic technique that greatly decreases drug production risks and costs. In this emergency, because of the availability of vast quantities of protection, pharmacodynamics, tolerability, pharmacokinetic, and clinical evidence on the current medication, it shortens the time period between finding a potentially beneficial medication and treating the patient. In this regard machine and deep learning can play an important role in designing new drugs. Drug repurposing is often serendipity result, it may likewise be the result of the detection of target similarities between various diseases, or experimental of drug screening, or the intervention of prevalent, pathogenetic mechanisms between various illnesses, similar to the scientific rules that motivated the previous-described repurposing experiments that are underway worldwide for curing COVID-19. Drug repurposing is a hypothesis-driven method that takes advantage of using big data to classify medications for the treatment of diseases or symptoms linked to diseases.

Deep learning can be used with data related to biological clinical characteristics, drug targets, pharmacological responses, and even off-target drugs to provide unexpected insights to understand COVID-19 pathology, symptoms, and potentially, medication identification. Deep learning may be possible to produce a hypothesisdriven, computer-assisted drug repurposing aimed for (i) reducing infection and replication of the virus; (ii) comparing adverse symptoms of the infection; (iii) understanding positive or negative interactions between treatments; (iv) identifying susceptibility mechanisms of the virus infection; and (v) predicting possible medication side effects against antiviral immune response, a possibility which may potentially lead to a worse clinical outcome. In this paper, BA is used as an optimizer with RNN to tackle the hyper parameters problems of RNN to build a new model to predict drug-target interactions for COVID-19.

The rest of this paper is structured as follows. Section II introduces the overview of COVI-19 disease with details about the RNN and BA. Section III describes the 
new prediction model of COVID-19 drug. In section IV, the experimental results have been presented and discussed. Section V concludes and presents the future work of this paper.

\section{Basics and Background}

\subsection{Coronavirus disease}

Coronaviruses are medical and veterinary importance viruses which include porcine epidemic diarrhea virus, transmissible gastroenteritis virus, and the human coronaviruses severe acute respiratory syndrome coronavirus (SARS COV) accountable for the epidemic in 2003, and Middle East respiratory syndrome coronavirus (MERS). Lots of new coronaviruses have been identified in the past ten years which infect an enormous variety of hosts from mammals to birds. Moreover, closely related coronaviruses were found in distant animals that suggest recent interspecies leaps (Masters, 2006). Such as influenza viruses with similar infectious features, they are single-stranded, lipoprotein-enveloped RNA viruses. Viral fusion proteins are possible vaccine and therapeutic targets and this is suggestive of the possibility of applying the kind of considerations addressed in this paper to a broader variety of viruses.

Until now, no recorded molecule has been found for treating COVID-19 patients. Nonetheless, there are ongoing studies that applied on using several anti-viral and immunomodulating or immunosuppressive medication has shown effectiveness on COVID-19 both in animal models, in vitro, and in small cases sequence (Robson, 2020). The emergency that the medical community faces in resolving the COVID-19 pandemic offers the basis for the use of drugs that have not yet been licensed and only have preliminary scientific evidence. Therapeutic protocols to date have included a mixture of anti-viral medications and preventive treatment.

In fact, there is no vaccine available for COVID-19 so far there is no antiviral drug recommended until now. The medicament is to minimize the symptomatic, and oxygen therapy is provided to patients with severe infection. Mechanical ventilation is required in cases of respiratory failure while hemodynamic support is necessary for managing septic shock. While research teams all over the world explore the main features, pathogenic, disease control, you should pay proper attention to each of the treatment options and the cross to meet the other. For example, vaccines for other diseases such as measles and rubella to give resistance crossed for COVID-19.

\subsection{Deep learning in drug design for COVID-19}

Currently deep learning as a subset of machine learning techniques can be utilized for drug discovery by analyzing the available COVID-19 data. It is helpful for medication delivery design especially with a huge amount of drug data. This technology is utilized to speed up drug testing in real-time, where normal testing takes a lot of time and thus helps to greatly speed up this process, which a human might not 
be able to do (Haleem et al., 2020; Biswas et al., 2019). Moreover, it can assist in identifying beneficial drugs for treating COVID-19 patients. It has become a paramount instrument for the development of diagnostic test designs and vaccination (Sohrabi et al., 2019; Chen et al., 2020; Bobdey et al, 2020). Furthermore, deep learning can be much faster than normal techniques in designing vaccines and therapies and is likewise useful in clinical trials during vaccine development (Robson, 2020; Beck et al., 2020).

Deep learning can be applied from a scientific and computational point of view, based on various methodologies and data types. Data types involve the properties of physicochemical, drug chemical structures, known molecular targets, and kinds of omics data, like transcriptional responses induced by drugs, or metabolic simulations. The methodologies range from traditional statistical methods to modern machine learning techniques.

To use the advantages of deep learning methods effectively, RNN is used in this paper. Different from previous studies in drug design, we propose a new method based on RNN and BA to overcome the hyper parameters problem of RNN.

\subsection{Recurrent Neural Network (RNN)}

$\mathrm{NN}$ is yet a different representative form of deep learning architecture. RNN has been widely used and achieved great success in NLP, with a particular emphasis on handling sequence data. RNN differs from standard neural feedforward networks (FNNs) that adopt the feedforward architecture. There is no connection between hidden nodes in the same layer in standard FNNs but only between nodes in adjacent layers. One of the major FNNs shortcomings is that they can not deal with sequence problems due to the output is not only concerning to recent input information but also to previous information, such as machine translation, for example (Barman et al., 2018). Yet, RNN can handle consecutive information by (1) inserting guided cycles into its net; (2) associating the adjacent hidden nodes with each other; (3) catching the measured information from previous time periods; and (4) saving it for the next step. The one-way data flux from input parts to output parts, moving during every hidden consecutive part. St reflects the phase $\mathrm{t}$ transition states, which refers to the memorial units in the net including the whole information derived from the sequence's prior data. In this step ( $\mathrm{t}$ ), the output from the output units is associated only with the state of the transition at that moment (St) (Barman et al., 2018). Each hidden layer with guided cycles is unfolding and processing in the RNN as a classical NN which shares the same weight matrices U, V, W in every layer. RNNs are plentifully variated. Among those RNN architectures, LSTM is considered the most common and widely utilized architecture in NLP today. Through NLP, LSTM is often paired with a distributed word embedding representation, which is accomplished by testing the statements and marking the part of the speech. 


\subsubsection{LSTM Networks}

Long Short-Term Memory Networks are a special type of RNN that can learn longterm dependencies. All recurrent neural nets take the form of a neural network repeating modules chain which has a very simple framework in standard RNNs, for instance, a single tanh layer. Moreover, LSTMs also have this chain-like framework but there is a different structure in the repeating module (Barman et al., 2018).

\subsection{Bat algorithm}

The Bat-inspired Algorithm is a heuristic algorithm proposed in (Lai et al., 2020). The Bat-inspired Algorithm has the advantages of rapid convergence, distribution potential, and parallelism. It is an efficient approach for the search for optimal global solutions. Researchers have been thoroughly investigating the BA's broad application potential and further improving the BA-based algorithm. The BA has greater convergence than other ones, and when approaching optimality the discovery and exploitation can be well balanced. Furthermore, the BA can solve restricted optimization problems easier. The BA is used in its current applications to refine solutions from an exact model (Yang, 2010).

For every bat, the position $x_{i}^{t}$ and velocity $v_{i}^{t}$ at iteration $t$ is calculated as

$$
\begin{gathered}
f_{i}=f_{\min }+\left(f_{\max }-f_{\min }\right) \cdot \beta \\
v_{i}^{t+1}=v_{i}^{t}+\left(x_{i}^{t}-x_{*}\right) \cdot f_{i} \\
x_{i}^{t+1}=x_{i}^{t}+v_{i}^{t+1}
\end{gathered}
$$

where $x_{*}$ is the recent global best location between the whole locations of the bat. Moreover, $\beta$ is a random vector between $[0,1]$. Here $f_{\max }$ and $f_{\min }$ depend on the domain size of the problem. A new solution for the local search is calculated as

$$
x_{\text {new }}=x_{\text {old }}+\varepsilon A_{\text {mean }}^{t}
$$

where $x_{\text {old }}$ is the recent best solution, $\varepsilon$ is a random number between [-1, 1], and $A_{\text {mean }}^{t}$ is the average loudness at time t.

The loudness $A_{i}$ and pulse emission rate are updated as

$$
\begin{gathered}
A_{i}^{t+1}=\alpha A_{i}^{t} \\
r_{i}^{t+1}=r_{i}^{0}[1-\exp (-\gamma t)]
\end{gathered}
$$

where $\alpha$ and $\gamma$ are constant for any $0<\alpha<1, \gamma>0, \mathrm{r} \in[0,1], A_{i}^{t} \rightarrow 0, r_{i}^{t} \rightarrow$ $r_{i}^{0}$, as $t \rightarrow \infty$.

\section{The Proposed Model for COVID-19 Drug}

The suggested model is an extremely efficient method to develop a group of sample candidate drugs using RNN model with BA which is applied to optimize the model parameters of RNN and then using the corona virus as a target, bind all the sample candidate drugs to it and find out the one with the best binding affinity. The drug with the best binding affinity will be a potential cure for the virus (Tang et al., 2020; 
Serafin et al., 2020; Shaha et al., 2020; Perricone et al., 2020). To the suggested algorithm steps are summarized in four main phases: (a) data preparation phase, (b) hyper-parameters optimizing phase, (c) learning phase, (d) fine-tuning for specific ligand subsets. Extra specifics for every phase are illustrated in the next sections.

The proposed approach is received as shown in Figure 1 input parameters related to a specific problem, like training data to be applied and parameters related to the RNN structures to be created, like the maximum number of particle layers through initialization. The algorithm shows the public framework for the proposed RNN- BA. In this algorithm, the best global particle relies in the selected blocks at the BA following the Bibliotheca Alexandrina. Therefore, there isn't need to manually optimize the parameters of all block. Moreover, parameter optimization does not start again, as good masses move to the following generation at the form of the best universal particle. Currently, particle evaluation requires only a restart at each iteration, but the proposed algorithm guarantees good block retention. Figure 1 shows the flowchart of the proposed algorithm using RNN-BA to classify the given data set.

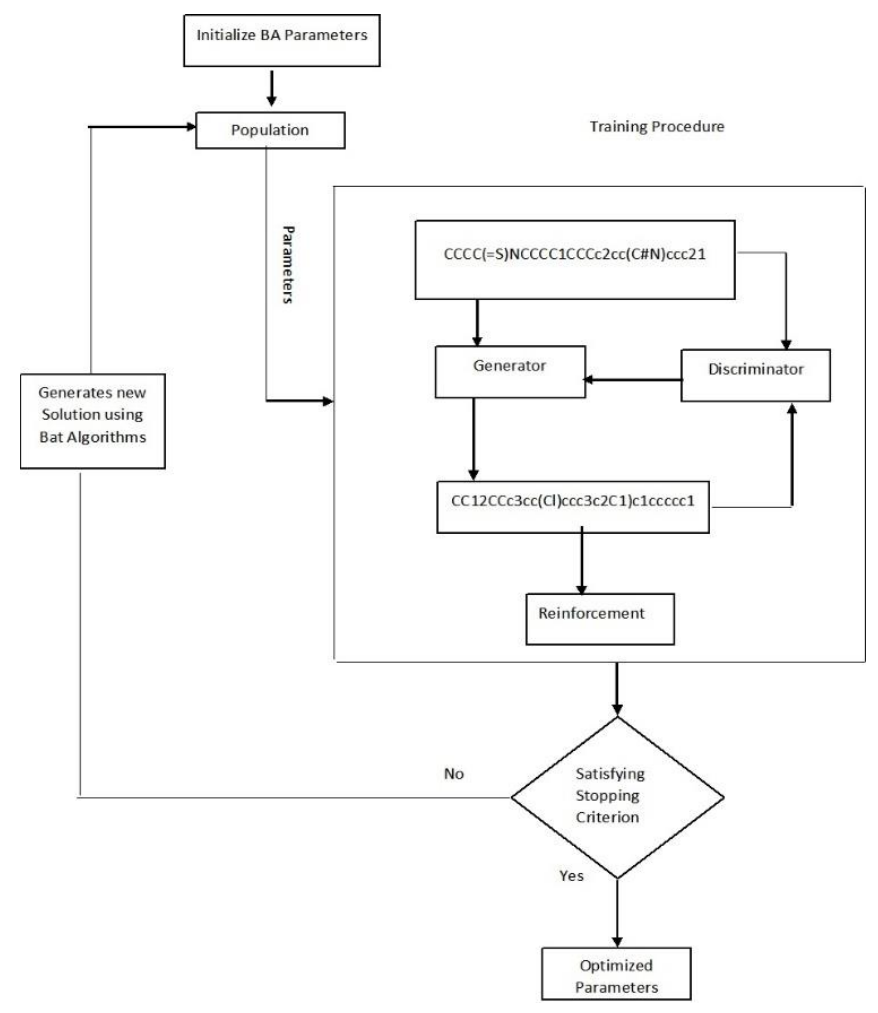

Fig. 1: The flowchart of the proposed algorithm using RNN-BA

\subsection{Dataset description}

The dataset which is used to train and evaluate the proposed approach is selected from a total of 677,044 SMILES. The dataset then preprocessed for eliminate duplicates, 
stereochemical information, and salts. Furthermore, the pre-processing filter of nucleic acids and peptides of the long-existing outside the space of the chemical which we sought to obtain samples from it. The training of the RNN, in the end, the 541, and 555 series SMILES, and lengths from 34 to 74 characters SMILES (tokens). Some samples of representative SMILES characters is given in Figure 2.

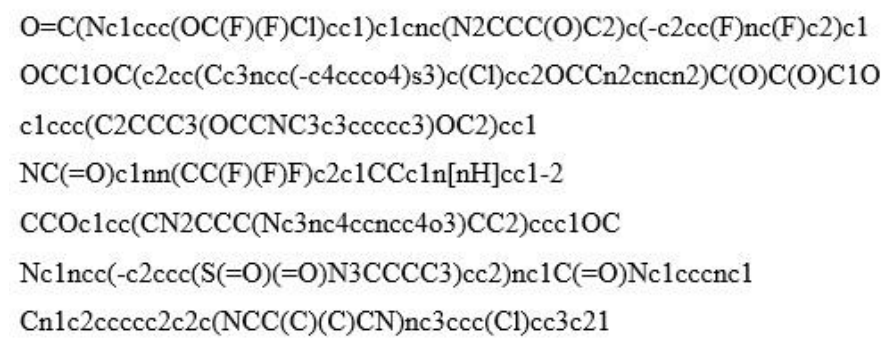

Fig.2: Samples of representative SMILES characters

\subsection{Data preparation phase}

The SMILES should clean to remove salts, duplicates and stereo-chemical information. The molecular matrix or the objective was set to Solubility which indicates how likely a molecule can mix with water. The SMILES were put into the model and trained.

\subsection{Hyper-parameters optimizing phase}

Often leads to choose the optimal parameters of the structure of the neural network to make a difference between expected performance and advanced performance. However, don't be posting only a few of the parameters and design options that should be evaluated or tested which makes the optimization of the parameters of excessive often Art black requires the experiences of experts.

The model represents the RNN set of parameters that allows parameter model RNN. The proposed method using model RNN for genetic by finding the optimum values to address the RNN with the assistance of the library of Alexandria so as to reduce the error of the training to a minimum. Initially, the parameters were initialized to BA (number of bats, loudness, and frequency, the maximum number of repeats, position, speed, and pulse rate). In the proposed model, the BA supplies the GAN classifier with $C$ values and the kernel parameter (i.e. in the RBF kernel or $d$ in the polynomial kernel) for the GAN training using the training set.

BA parameters. For initialization parameters for BA algorithms, we used the values shown in Table 1. Created with specific parameters, BA is used to find the optimal values for interval number, batch size, learning rate, and the number of neurons in the first hidden layer of RNN. 
Table 1. The used parameter values for BA algorithm

\begin{tabular}{|c|c|}
\hline Parameter & BA \\
\hline Dimension of the problem & 4 \\
\hline Population size & 40 \\
\hline Number of generations & 100 \\
\hline Loudness & 0.5 \\
\hline Pulse rate & 0.5 \\
\hline Min. frequency & 0 \\
\hline Max. frequency & 2 \\
\hline Lower bound & 0 \\
\hline Upper bound & 1 \\
\hline & \\
\hline
\end{tabular}

\subsection{Learning Phase}

Two approaches for RNN training were tested. The first approach was to split every entry into nested windows of some length 1 and expect the token $1+1$ st for each window (Model 1). The loss was determined from the $1+1$ st token probability. As shown in Figure 3a, the second training method that we used pads each string entered into $\mathrm{n}$ of tokens, where $\mathrm{n}$ is the length of the longest SMILES string. The algorithm expects the following token inside the string for each token (Model2). The loss in all molecules was averaged over all of the target tokens. We've only covered the RNN Code case ' $G$ ' and sample the next character of the distribution is expected when sampling from the model trainer using Method 2. This was the following message associated with the 'G.' every time we raise another available letter in the sequence that was previously created so that the creation of the token 'E' (Figure $3 \mathrm{~B}$ ). The parameter has been added to the temperature added to the function of the softmax sampling of letters from the model (Figure $3 \mathrm{C}$ ). The lead temperature associated with the sampling of the diversity of the structure of the bigger structures of the molecular region but it decreases the safety of the SMILES of the appropriate chemically while the low temperature to the diverse structure of less, but the predictions more conservative.

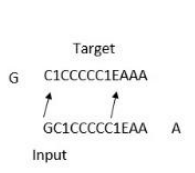

(a) Training

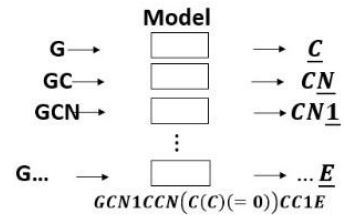

(b) Sampling

$$
\begin{gathered}
L=\sum_{i=1}^{k} y_{i} \log (\hat{y}) \\
P\left(y_{i}\right)=\frac{\exp \left(y_{i} / T\right)}{\sum_{j=1}^{n} \exp \left(y_{j} / T\right)}
\end{gathered}
$$

(c)

Fig. 3: a) Training procedure b) Sampling procedure c) loss error L and the softmax functions 


\subsection{Fine-tuning for subsets ligand-specific}

After training the model on the producing strings of SMILES is valid, we tried to adjust the model with fine-tuning through furthermore training on smaller subsets of the compounds selected. The goal was adapting the model to producing a series SMILES with similarity higher with the dataset that focuses on the target. Ligands were drawn for the target from the ChEMBL dataset. The pre-treated of the group was preprocessed by removing of salt and stereochemistry, and the selection of eighty percent of the central particles through the length of the strings of SMILES corresponding canonical. After all of each training period, the sample-set was created.

\section{Experiment Results, Discussion and Analysis}

The deep learning model that used to this experiment is a adjust version of GAN (Generative Adversarial Network). The GAN model has two neural networks, which is a Generator and Discriminator.

The Generator's main objective is to generate fake samples which so closely look at the true data/distribution that the discriminator can't distinguish between the true and the fake data. As for the Discriminator, it discriminates the input data and classifies whether it is forming the true data sample/distribution, or is a fake sample generated by the Generator. It is initially trained for true samples of the labeled data. The two networks are working against every other to try that work for proving themselves better and that main goal is to generate data points such as some of the data points consisting of the training data.

The Generator G samples x from a distribution Psynth (Fake Data), generated with random noise $\mathrm{z}$, while the discriminator D looks at samples, either from Psynth (Fake Data) or Pdata (Real Data) and attempts to classify their identity y as either real or fake.

The power and stability of the GAN are limited by various problems. While training GAN, you will understand, the discriminator part is more powerful than its generator counterpart. The generator would thus fail to effectively train who will turn result in a larger loss in your GAN training process. But if the discriminator is too lenient, it would literally permit the generation of any image. This entire idea will thus remain useless to your GAN. To solve these problems integrate the reinforcement learning to the generator training so that it generates the required outputs by updating the generator parameters with the policy gradient.

We treated the Generator $\mathrm{G}$ as an agent in an RL game with a policy gradient in which we consider states $\mathrm{s}$, actions from an action space $\mathrm{A}$, and a reward function $\mathrm{Q}$. A state st is a partial sequence of characters X1:t which is already generated. We have an action space A that includes all possible characters to choose from for the next $\mathrm{xt}+1$ character. Next, a reward function $\mathrm{Q}(\mathrm{s}, \mathrm{a})$ which represents the expected reward for taking action an in state $\mathrm{s}$. Each episode is the completion of a fully generated fixed-length T sequence which is rewarded with the RT (X) function. The stochastic 
policy of the agent is given by $(\boldsymbol{y t} \mid \boldsymbol{Y} \mathbf{1}: \boldsymbol{t}-\mathbf{1})$ and we want to maximize the long-term reward $\mathrm{J}(\mathrm{w})$ expected as in equation (7).

$$
j(\theta)=E\left[R_{T} \mid s_{\theta}, \theta\right]=\sum_{x_{1} \in X} G_{\theta}\left(x_{1} \mid s_{\theta}\right) \cdot Q\left(s_{\theta}, x_{1}\right)
$$

It should be noted that molecular measures such as drug similarity and the likeness of a natural product were used to increase the reward by improving the generator to generate molecules similar to the primary data distribution. Then the discriminator analyzes the generated molecules and the reward scale. Then you improve the generator and train it to deceive the distinguished.

The above steps are where the pre-training phase formed the first half of the training. In the last half of the training, a policy hierarchy is used to train both the generator and the distinguished. The loss is calculated for each character generated, and the model is updated.

Policy gradient loss is calculated in the case of the generator. After that, the generator is optimized, and all parameters are updated. The policy function calculates the output sequence Log SoftMax that gives rewards, destinations, and sequence length. Its result is negative because we want to reduce the loss but maximize the policy gradients as in equation (8).

$$
L=-Q(s, a) \log \left(G\left(y_{t} \mid Y_{1: t-1}\right)\right)
$$

where $\mathrm{Q}(\mathrm{s}, \mathrm{a})$ expected reward for an action a in state s and $\boldsymbol{G}(\boldsymbol{y t} \mid \boldsymbol{Y 1 : t}-\mathbf{1})$.

The experiment performs in that paper is greatly affected by the efforts. Firstly, the Deep Reinforcement Learning model is applied to generate the possibility of candidates'. The SMILES should be cleaned to delete salts, duplicates, and stereo chemical information. The molecular matrix or the objective was set to Solubility who indicates how probable a molecule can be mixed with water. The SMILES were put into the model and trained. After about 9 hours of training, with a $\lambda$ of 0.2 and epochs of 240 and a sample set of 6400, 10 good sample as in Figure 4 SMILES were generated and The Solubility or LogP of these samples is 0.7098 .

$\mathrm{COC}(=\mathrm{O}) \mathrm{c} 1 \mathrm{cccc}(\mathrm{Cl}) \mathrm{c} 1 \mathrm{OC}(\mathrm{O}) \mathrm{CO}$
$\mathrm{O}=\mathrm{C}(\mathrm{CCOCCCCCCCCCCF}) \mathrm{CCCCCCCCC}$
$\mathrm{O}=\mathrm{C} 1 \mathrm{CCCN}(\mathrm{CCCNC}(=\mathrm{O}) \mathrm{c} 2 \mathrm{ccoc} 2) \mathrm{CC} 1$
$\mathrm{COCCNCCCCCCCC} 1 \mathrm{nc} 2 \mathrm{cc}(\mathrm{O}) \mathrm{c}(\mathrm{F}) \mathrm{cc} 21$
$\mathrm{O}=\mathrm{C}(\mathrm{NCc} 1 \mathrm{ccncc} 1) \mathrm{C} 1 \mathrm{CN} 2 \mathrm{CC}=\mathrm{CC} 2 \mathrm{CC} 1$
$\mathrm{O}=\mathrm{C} 1 \mathrm{C}(\mathrm{Cl})=\mathrm{CC} 2(\mathrm{CCCc} 3 \mathrm{ccccc} 3) \mathrm{CC} 2=\mathrm{C} 1$
$\mathrm{CCCCCCC}(\mathrm{C}=\mathrm{CCCCCCCN}) \mathrm{CC}(=\mathrm{O}) \mathrm{O}$
$\mathrm{Cc} 1 \mathrm{cncc}(\mathrm{Cc} 2 \mathrm{cccc} 2) \mathrm{c} 1 \mathrm{C}(=\mathrm{O}) \mathrm{CCCCO}$
$\mathrm{COc} 1 \mathrm{ccc}(\mathrm{C}=\mathrm{Cc} 2 \mathrm{cccnc} 2) \mathrm{c} 2 \mathrm{ccccc} 12$
$\mathrm{CC} 12 \mathrm{CCCCC} 1 \mathrm{CCC}(\mathrm{CC}(\mathrm{C}) \mathrm{C}=\mathrm{S}) \mathrm{CCCC} 2$

Fig 4: Example of good samples 
Other molecular matrixes for the 10 good samples were also listed, as displayed in Table 2. The novelty of the sample drugs which shows how molecularly distinct these samples drugs are from the other candidate drugs is seen to have a high value of 1 . The synthesizability which shows or indicates how hard (0) or easy (0.9997) it is to synthesize these 10 good sample molecules has a value of 0.6269 .

Table 2. The molecular matrixes

\begin{tabular}{|c|c|}
\hline Novelty & $\mathbf{0 . 9 9 9 7}$ \\
\hline hard_novelty & 0.9997 \\
\hline soft_novelty & 0.9998 \\
\hline diversity & 0.6269 \\
\hline conciseness & 0.9937 \\
\hline solubility & 0.6683 \\
\hline naturalness & 0.6350 \\
\hline synthesizability & 0.7278 \\
\hline
\end{tabular}

After that 10 good sample candidates' drugs were collected, there were all bonded to the coronavirus during the PyRX software. The binding affinities of each of the samples were listed with the results displayed by the figure below in Figure 5.

\begin{tabular}{|r|l|r|r|r|}
\hline 1 & Ligand & Binding Airmsd/ub & $\mathrm{rmsd} / \mathrm{lb}$ \\
\hline 2 & 6lu7_C18H15ClN4O2_uff_E=471.56 & -7.3 & 0 & 0 \\
\hline 3 & 6lu7_C23H20N2O_uff_E=2008.31 & -6.9 & 0 & 0 \\
\hline 4 & $6 l u 7$ C21H18FNO_uff_E=469.56 & -6.8 & 0 & 0 \\
\hline 5 & 6lu7_C18H15CIN4O2_uff_E=471.56 & -6.8 & 6.651 & 3.671 \\
\hline 6 & 6lu7_C21H18FNO_uff_E=469.56 & -6.5 & 7.139 & 2.851 \\
\hline 7 & 6lu7_C21H18O2_uff_E=353.61 & -6.4 & 0 & 0 \\
\hline 8 & 6lu7_C21H18FNO_uff_E=469.56 & -6.3 & 20.923 & 18.893 \\
\hline 9 & 6lu7_C21H18FNO_uff_E=469.56 & -6.2 & 0 & 0 \\
\hline 10 & 6lu7_C18H15CIN4O2_uff_E=471.56 & -6.2 & 6.017 & 3.844 \\
\hline
\end{tabular}

Fig.5: Bonding affinities of candidate drugs

From Figure 5, the drug with the highest binding affinity (Most negative value) is $\mathrm{C} 18 \mathrm{H} 15 \mathrm{ClN} 4 \mathrm{O} 2$ also known as Olutasidenib. This is the best candidate drug that has the potential of being an anti-viral cure to the coronavirus. Olutasidenib is an active, selective mutant Isocitrate dehydrogenase (IDH) 1 inhibitor for treating acute myeloid leukemia. The results produced through the experiments conducted above tend to convey that this drug could also be a potent cure for the coronavirus.

Table 3 reports the accuracy of our approaches against other models. We can see RNN- BA get better the performance over models RNN, which demonstrates the effectiveness of the RNN- BA model. Compared with other models, RNN- BA gains the best performance in 8 tasks and show very competitive results on the others. 
Table 3: The results obtained from the comparative models between RNN model and RNN- BA model

\begin{tabular}{|c|c|c|}
\hline Epoch number & RNN & RNN- BA \\
\hline Epoch 1 & 94.09 & $\mathbf{9 7 . 9 2}$ \\
\hline Epoch 2 & 93.89 & $\mathbf{9 6 . 0 8}$ \\
\hline Epoch 3 & 93.83 & $\mathbf{9 6 . 9 7}$ \\
\hline Epoch 4 & 92.89 & $\mathbf{9 6 . 8 8}$ \\
\hline Epoch 5 & 92.26 & $\mathbf{9 5 . 9 6}$ \\
\hline Epoch 6 & 90.01 & $\mathbf{9 3 . 1 4}$ \\
\hline Epoch 7 & 91.22 & $\mathbf{9 5 . 0 6}$ \\
\hline Epoch 8 & 93.15 & $\mathbf{9 6 . 0 8}$ \\
\hline
\end{tabular}

In Figure 6, we illustrate the curves of the best accuracy model found by RNN BA on the Convex dataset for 60 epochs comparative by RNN model. We can see the accuracy of our model raised over the RNN model which means that the proposed RNN- BA is truly adequate for finding good models for a given image classification dataset (Batioua et al., 2018; Qiao et al., 2017).

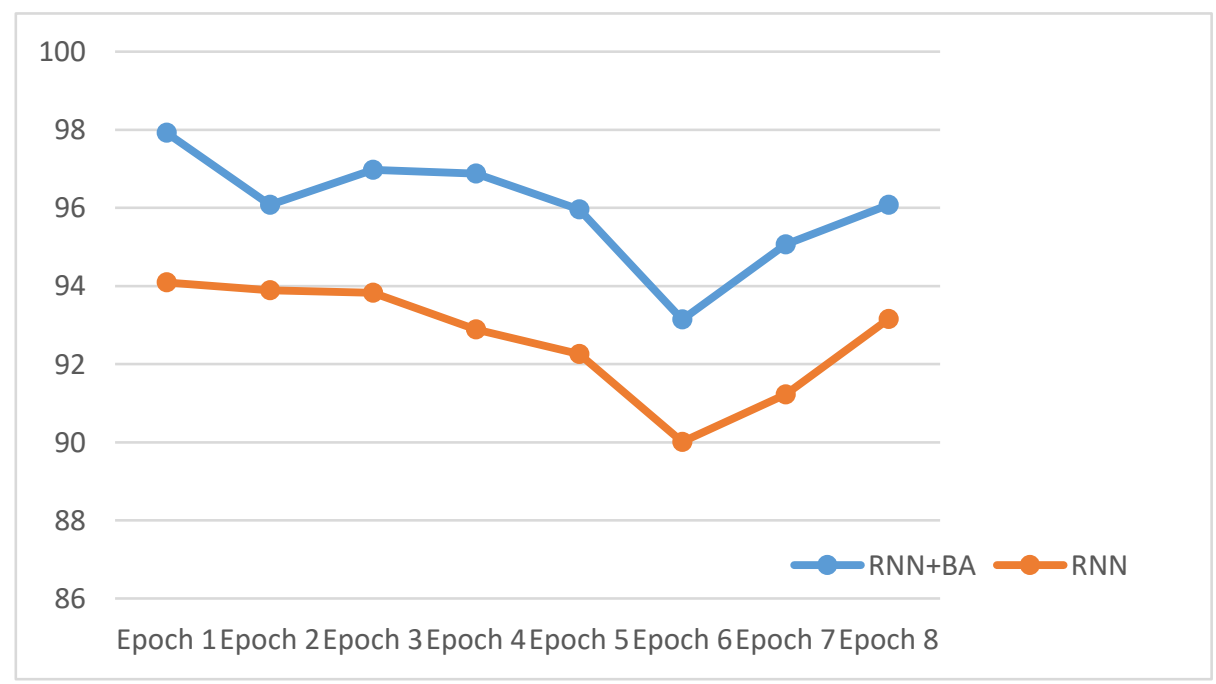

Fig. 6: Accuracy curves for comparative between the RNN model and RNN-BA model

\section{Conclusion and Future Work}

A viral disease of the coronary (COVID-19) is a severe viral infection of pathogenic caused by SARS-CoV-2. Currently, the cause of COVID-19 in health concern globally. Supposed to COVID-19 has zoonotic origin depends on a large infection people who have been exposed to the wet market in Wuhan City, China Given the paramount importance of the epidemic and the current caused by the COVID-19, due to the fact that scientists agree that there is no medication specific are proposed to the treatment alternatives possible through the reorganization of the pharmaceutical. In 
this paper, BA is used in this paper to optimize the model parameters of RNN and then using the corona virus as a target. The drug with the best binding affinity will be a potential cure for the virus. The used dataset in this paper is utilized to train and evaluate the proposed approach is selected from a total of 677,044 SMILES. The dataset then preprocessed to delete the repeat, salts, and chemical information vacuum. Also, preprocessing of the nucleic acids and peptides long that fall outside the chemical space that which we sought to obtain samples from it. The promising experimental results of the proposed model in comparison with RNN showed high level of perdition and performance.

Further evaluation of the efficacy of current antiviral drugs in clinical applications is needed. Also, we encourage a more complex investigation of the antiviral effect of these molecules against SARS-CoV-2

\section{References}

Wang, M., Cao, R., Zhang, L., Yang, X., Liu, J., Xu, M., Shi, Z., Hu, Z., Zhong, W. and Xiao, G., 2020. Remdesivir and chloroquine effectively inhibit the recently emerged novel coronavirus (2019-nCoV) in vitro. Cell research, 30(3), pp.269-271.

Duddu, P., 2020. Coronavirus outbreak: Top coronavirus drugs and vaccines in development. Available from:. clinicaltrialsarena.com/analysis/coronavirus-merscov-dru gs/, accessed on February, 13.

Ifiok Okokon Charles., 2020 Anti-Viral Cure of COVID-19 Using Deep Reinforcement Learning., https://github.com/Ifiokcharles.

Barman PP, Boruah A. (2018). A RNN, based Approach for next word prediction in Assamese Phonetic Transcription. Procedia computer science, 143,117-23.

Batioua I., Benouini R., Zenkouar K., Zahi A., Image classification using separable invariants moments based on racah polynomials, Procedia Comput. Sci. 127, 320327.

Beck B.R., Shin B., Choi Y., Park Sungsoo., Kang K. (2020), Predicting commercially available antiviral drugs that may act on the novel coronavirus (SARSCoV-2) through a drug-target interaction deep learning model, Computational and Structural Biotechnology Journal, 18, 784-790.

Biswas K., Sen P. (2020). Space-time dependence of coronavirus (COVID-19) outbreak. arXiv preprint arXiv, 03149. 
Bobdey S., Ray S. (2020). Going viraleCOVID-19 impact assessment: a perspective beyond clinical practice. J Mar Med Soc, 22(1), 9.

Chen S., Yang J., Yang W., Wang C., B€arnighausen T. (2020). COVID-19 control in China during mass population movements at New Year. Lancet. https:// doi.org/10.1016/S0140-6736, 30421-9.

Haleem A., Vaishya R., Javaid M., Khan IH. (2019). Artificial Intelligence (AI) applications in orthopaedics: an innovative technology to embrace. J Clin Orthop Trauma. https://doi.org/10.1016/j.jcot.2019.06.012.

Lai C.C., Shih T.P., Ko W.C., Tang H.J., Hsueh P.R. (2020). Severe acute respiratory syndrome coronavirus 2 (SARS-CoV-2) and coronavirus disease-2019 (COVID-19): The epidemic and the challenges. Int. J. Antimicrob. Agents. 55, 1059249.

Masters P.S. (2006). The molecular biology of Coronaviruses, Adv. Virus Res. 66, 193-292.

Perricone C., Triggianese P., Bartoloni E., Cafaro G., Bonifacio A.F., Bursi R., Perricone R., Gerli R. (2020). The anti-viral facet of anti-rheumatic drugs: Lessons from COVID-19, Journal of Autoimmunity, Available online, 102468.

Robson B. (2020). Computers and viral diseases. Preliminary bioinformatics studies on the design of a synthetic vaccine and a preventative peptidomimetic, antagonist against the SARS-CoV-2 (2019-nCoV, COVID-19) coronavirus. Computers in Biology and Medicine, 119, 103670.

Serafin B. , Bottega A., Foletto V. S., da Rosa T. F. , H' orner A. , H “' orner R. (2020), Drug repositioning an alternative for the treatment of coronavirus COVID19, International Journal of Antimicrobial Agents, Available online, 105969.

Shaha B., Modi P. and Sagar S. R. (2020). In silico studies on therapeutic agents for COVID-19: Drug repurposing approach, Life Sciences, 252, 117652.

Sohrabi C., Alsafi Z., O’Neill N., Khan M., Kerwan A., Al-Jabir A., Iosifidis C., Agha R.(2020). World Health Organization declares global emergency: a review of the 2019 novel coronavirus (COVID-19). Int J Surg.

Tang H., Sun W., Yu H., Lin A. and Xue M. (2020), A multirobot target searching method based on bat algorithm in unknown environments, Expert Systems With Applications, 141, 112945. 
Yang X.S. (2010), Nature Inspired Cooperative Strategies for Optimization (NISO 2010), Springer Berlin Heidelberg, Berlin, 65-74.

Qiao T., Retraint F., Cogranne R.., Thai T.H., Individual camera device identification from JPEG images, Signal Process., Image Commun, 52 (3), 74-86. 